\title{
Anterior repositioning splint treatment consideration for cases of disc displacement with reduction
}

\author{
'Steven Alexander Tjang, ${ }^{2}$ Ira Tanti \\ ${ }^{1}$ Prosthodontic Residency Program \\ ${ }^{2}$ Prosthodontic Department \\ Faculty of Dentistry, Indonesia University \\ Jakarta, Indonesia \\ Corresponding author: Ira Tanty, email: iratanti@ymail.com
}

\begin{abstract}
The focus of this literature review is to explore the process of disc displacement with reduction (DDWR) and how the treatment using anterior repositioning splint(ARS) can positively affectpatients who are diagnosed with DDWR. The use of MRI as the gold standard of TMJ study allows current researcher to re-consider the common theory whereby DDWRultimately progresses into disc displacement without reduction or other advancedinternal derangement process. The use of ARS can produce resolution of pain in patients who are experiencing DDWR with joint pain. However, the use of ARS is shown to be effective on short term only, and its use must be accompanied by other modalities such as physical self-regulation (PSR). The lack of gold standard for the treatment of DDWR prompts healthcare worker to provide patients with conservative treatment before offering more advanced and sur-gical procedure, both of which carries additional unwanted risk. Overall, the use of ARS is shown to be very effective and non-invasive.
\end{abstract}

Keywords: disc displacement with reduction, anterior repositioning splint, TMD

\section{INTRODUCTION}

Temporomandibular disorder (TMD) is a group of conditions that involve masticatory muscles, temporomandibular joint (TMJ) and the surrounding structures. Generally, the term intercapsular disorders involves muscles of mastication, whilst intracapsular disorder involves temporomandibularjointcomplex. From the myriads of intracapsulardisordervariations, disc displacement with reduction (DDWR) conditions comprises of $41 \%$ of the diagnoses of TMD that is often founded by clinician. ${ }^{1}$ Observation of the condition of DDWR under magnetic resonance imaging (MRI) show articulardisc position located more anteriorly than normal during maximum intercuspation. During mouth opening, articular disc will return to a normalposition, whereby the returning motion of condyle to normal position from anterior position creates the clicking sound. DDWR can be accompanied with pain or without pain. A recent publications show $31 \%$ of patients has no signs of painful condition. ${ }^{2}$

Correlation between pain and TMD is still a matter of debate. Okeson explained that intracapsular disorder can occur on patients with orthopedically unstable TMJ as well as unfavorable masticatory force. However, notall orthopedically unstable TMJ will lead to intracapsular disorder, and therefore therapy is not always indicated. ${ }^{1}$ The high prevalence of DDWR among general population causes confusion for both patient and clinicians in terms of the risk factor associated with the progression on DDWR. A literature review is important to determine whetherDDWR treatment will be needed and/or the degree of treatment that can be provided for patient. ${ }^{2}$

The use of anterior repositioning splint (ARS) has been known as one of the treatment alternatives for DDWR with a good success rate. A shortterm use of ARS has been shown to be able to reduce painful symptom associated with DDWR. However, several researchers found that the longterm use of ASR can cause relapse to DDWR, making articular disc condition return to a state before treatment. ${ }^{2,3}$

This literature review will revisit DDWR, its progression, the need to alleviate the condition and ultimately to determine whether the use of ARS can become a routine procedure to treat and resolve DDWR that often becomes patient's chief complaint.

\section{METHODS \\ Focused questions}

Using patient, intervention, comparison, outcome (PICO) format, the following criteria were framed for this literature search, that is $P$ : patients with DDWR that seeks treatment for their condition due to the presence of pain and/or clicking sound; I: patients instructed to use ARS as a form of treatment for DDWR; C: comparing the position of articular disc within TMJ complex before, during and after treatment with or without the use of ARS. Pain and clicking evaluation before 
and after treatment with ARS compared to without the use of ARS. Comparisons performed using $\mathrm{MRI}$; O: effectiveness of ARS with the following outcome that is pain resolution, clicking sound resolution and resolution in disc position within the TMJ complex.

\section{Search strategy method}

Using electronic search of three databases, MEDLINE (Via PubMed), Cochrane Library and Scopus, was performed to identify the relevant literature. Articles that are published in the last 10 years, from January 2010 to December 2019, were considered. The following combination of keywords was applied ((ARS [All Fields] OR anterior repositioning appliance[All Fields]) AND (DDWR [All Fields] OR TMD [All Fields] OR TMJ dysfunction [All Fields] OR TMJ disease [All Fields]) AND (MRI [All Fields] OR MRI [All Fields])).

Additionally, manual search of relevant articles was performed. Articles published between the year 2010 through the latest, which is 2019, was performed in the following journals: Journal of Cranio-Maxillofacial Surgery, Journal of Oral Rehabilitation, Scientific Reports, Journal of Applied Oral Science.

\section{Inclusion and exclusion criteria}

The following inclusion criteria were applied: any case series, prospective, retrospective, cohort studies, controlled clinical trials, or randomized clinical trials with five or more patients included, full text in English, and a minimum of patient followup performed after treatment.

The following exclusion criteria were applied: in vitro studies and animal studies

\section{RESULT}

Using the search strategy, 14 articles from Cochrane Library, 6 articles Scopus, and 5 articles from MEDLINE via PubMed. Using the inclusion and exclusion criteria, 6 articles were selected to be part of inclusion criteria.

Article by Liu et al evaluated the effect of bite position when differentsplint treatments are used to a sample of 37 subject with a mean age of 18.8 \pm 4 .3years old. Maximum intercuspation was used as control, while ARS is being compared with stabilization splint. Measurement using MRI is performed by determining the disc-condyle angle during the use of the splint. In this study it was shown thatARS improves relationship between disc condyle more than stabilization splint and control. However, it is also shown that transitory posterior movement of the disc also occurred. Here the author mentioned that as soon as ARS is no longer being used, articulardisc will return to its displaced position. The long term effect of continual use of ARS is not being investigated.

Chantaracherd investigated moreabout the

Table 1 The 6 journals selected to be part of inclusion criteria

\begin{tabular}{|c|c|c|c|}
\hline Author & $\begin{array}{l}\text { Findings on pain } \\
\text { resolution }\end{array}$ & $\begin{array}{l}\text { Findings on clicking } \\
\text { resolution }\end{array}$ & $\begin{array}{l}\text { Findings on change in discal } \\
\text { recapture after the use of ARS }\end{array}$ \\
\hline Liu et $\mathrm{al}^{5}$ & No information on pain & $\begin{array}{l}\text { ARS resolve clicking sound, } \\
\text { but the sound returns after } \\
\text { no longer using ARS }\end{array}$ & $\begin{array}{l}\text { Disc recapture was more significant in } \\
\text { the use of ARS when compared to con- } \\
\text { trol and SA (stabilization appliance) }\end{array}$ \\
\hline $\begin{array}{l}\text { Chantaracherd } \\
\text { et } \mathrm{al}^{4}\end{array}$ & $\begin{array}{l}\text { Using characteristic pain in- } \\
\text { tensity }(\mathrm{CPI}), \text { ARS is statisti- } \\
\text { cally significant up to } 3 \text { mo- } \\
\text { nths after treatment end }\end{array}$ & $\begin{array}{l}\text { ARS use has no impact } \\
\text { on clicking sound }\end{array}$ & No information on discal position \\
\hline Ma et $\mathrm{al}^{6}$ & $\begin{array}{l}\text { Statistically significant } \\
\text { reduction in joint pain }\end{array}$ & $\begin{array}{l}\text { Statistically significant } \\
\text { reduction in joint clicking }\end{array}$ & $\begin{array}{l}\text { Disc recapture was statistically signifi- } \\
\text { cant in younger subject (early adoles- } \\
\text { cent) but not in older (late adolescent) }\end{array}$ \\
\hline Litko et $\mathrm{al}^{8}$ & $\begin{array}{l}\text { Statistically significant in } \\
\text { joint pain }\end{array}$ & $\begin{array}{l}\text { No information in joint } \\
\text { clicking, but patient with } \\
\text { limited mouth opening } \\
\text { show sign of improvement. }\end{array}$ & $\begin{array}{l}\text { No information on disc recapture, but } \\
\text { correlation is made between the } \\
\text { severity of disc displacement with } \\
\text { restriction in mouth opening. }\end{array}$ \\
\hline Xie et $\mathrm{al}^{7}$ & $\begin{array}{l}\text { No pain is reported after } \\
\text { the use of ARS }\end{array}$ & $\begin{array}{l}\text { No information in joint } \\
\text { clicking }\end{array}$ & $\begin{array}{l}\text { No information in disc recapture, butin } \\
\text { adolescent patientit shows mandibular } \\
\text { asymmetry for patient who does not } \\
\text { receive ARS after } 6 \text { months follow up }\end{array}$ \\
\hline Chen et $\mathrm{al}^{3}$ & $\begin{array}{l}\text { Pain resolves after treat- } \\
\text { ment with no recurrence }\end{array}$ & $\begin{array}{l}\text { Joint clicking sound com- } \\
\text { pletely disappeared du- } \\
\text { ring observation period }\end{array}$ & $\begin{array}{l}\text { Disc recapture is shown from the time } \\
\text { of ARS use and up to } 6 \text { months post- } \\
\text { treatment. After that period, disc recap- } \\
\text { ture only appears in } 40.6 \% \text { of subject }\end{array}$ \\
\hline
\end{tabular}


conventional pathway of the progressionofDDWR to disc displacement withoutreduction (DDWoR). The use of ARS is shown to alleviate pain in patient with DDWR, but patient without pain that do not use ARS is also shown to remain stable for years after observation. It seems that the progression of DDWR to DDWoR, as we previously believed, is notan absolute theory, because some people, especially older people, can experience DDWR without progressing to degenerative disease. Therefore, in this cross sectional studies there is not association between TMJ intra-articular status and TMD impact.

Ma etal tries to determine the efficacy of ARS in DDWR patient with class II occlusal relationship in 91 subjects with mean age of 15.7 years old. The observation was performed using MRI and images were taken before treatment (T0), after bite registration ( $\mathrm{T} 1$ ), at the end of treatment (T2), and 12 months after treatment (T3). In this study it was shown that the efficacy of ARS decreases over time. However, from clinical and MRI findings it can be concluded that ARS is an effective treatment modality in DDWR, especially for patients in early puberty.

Litko etal analyses the degree of DDWR with restriction in mouth opening; 191 patients: 148 women and 43 men ages 14-60 years old, that are diagnosed with DDWR were treated and found that the severity of DDWR from sagittal is statistically significant predictor in mouth opening. The association of disc displacement and TMJ internal derangement were made in this journal, and no clicking is mentioned during the trial.

Xie etal studies how DDWR affects mandibular asymmetry in adolescent patient. In this selfcontrol longitudinal study, craniofascial growth were recorded at least 6 months in 40 patients ages 10-20 years, and found that if DDWR is not treated, then the severity of mandibular asymmetry during growth increases. There is no mention on joint pain and clicking associated with DDWR.

The study by Chen et al examines the short and long term effect of ARS on disc condyle by metricanalysis by Draze-Enazmann Disccondyle angle using MRI. 32 TMJ were studied and MRI image were taken before the study, immediately post-insertion and 6 months after treatment. The study found that ARS has agood short term effect on disc condyle complex with relatively lower efficacy in long term of 6-months observation period.

When comparing results between the articles, many heterogenities of results are shown in terms of subject choice, methods of assessment, and factors to be considered. Some articles, such as Ma et al and Xie et al uses subject ages 12-18 years old to assess the treatment of DDWR on a subject that are still undergoing growth stage. This can produce different result when compared to older subject who are no longer growing. Moreover, some subjects use questionnaire as a form of assessment in terms of improvement, such as Chantaracherd et al and Litko et al, whereas other researchers use MRI as the gold standard of TMJ assessment. These differences need to be taken into account when analyzing the results that they have.

In general, these articles have similarity in conclusion especially 1) the use of ARS is an effective modality to treat DDWR. Results is statistically significant during and post-treatment. However, after 6 months, some patients have their condition relapsed back into DDWR. Therefore longitudinal studies are needed to further assess the efficacy of ARS; 2) the lack of treatment of DDWRdoesnotlead intofurtherdiscderangement disorder such as DDWoR or other degenerative disorder. These chains of causality must be investigated further.

\section{DISCUSSION \\ Disc displacement with reduction}

DDWR is known to be the most common internal derangement of TMJ condition in the majority of the population. ${ }^{6}$ When teeth occlude, articular disc is positioned more anterior than normal, and often reduced to normal position during mouth opening. This condition is thought to be caused by biomechanical pressure that the condyle receive against mandibula, which can cause progressive change in function and form. Various factors that potentially play a role in increasing the risk of DDWR can be summed into microtrauma and macrotrauma. Macrotrauma, such as automobile accidentora physical hit to lower jaw, can be easily found out during anamnesis. Microtrauma, however, can be undetected when examined with an inexperienced clinician. One of the example of microtrauma is bruxism ${ }^{2}$, and orthopedic instability thatcan happen in tooth arrangement that are unstable. It can also happen to Angle class II division two which is often found to have positive correlation with DDWR. Other condition, such as hypoxia reperfusion injury is also anotherform if microtrauma whereby overloading joint overtime can cause soft tissue degradation. Damage to collagen fibril causes reduction in collagen network, and secretion of synovial fluid from 
articular disc creates a more tender disc. This condition is also known as chondromalacia. When this condition left untreated, articular disc adhesion can occur at the superior compartment of articular disc, which contributes to DDWR. ${ }^{1}$

The progress of DDWR can be explained through elongation of discal collateral ligament. Thinning of the posterior border of articular disc canalso cause to displace articular disc anteriorly, which shifts condyle position to a more posterior position, closing in to retrodiscal tissue. This could potentiate into DDWR with painful symptoms due to aggravation of retrodiscal tissue. When patient opens their mouth, the motion of mouth opening cause changes in articular disc position. ${ }^{2,9}$

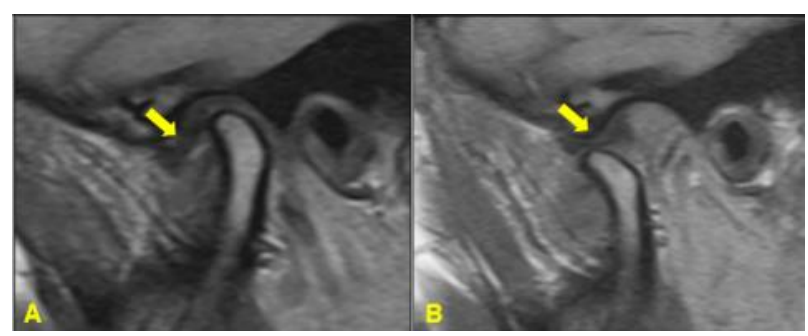

Figure 1 DDWR; A during maximum intercuspation, articular disc (yellow arrow) is located more anteriorly in its relation with TMJ's condyle fossacomplex; B during maximum mouth opening position, articular disc returns to normal position, where it articulates well in between condyle and articular eminence. ${ }^{2}$

The prevalence of DDWR is higher among women than men. It is speculated that the joint laxity of women's joint articulation along with higher intra-articular pressure is one of few probable causes of this discrepancy when compared to men. Furthermore, there are positive correlation between an increase in age and an increase in prevalence which is thought to be caused by the change in articularis disc dimension along with advancing age. ${ }^{2}$ Other researcher have found that anestrogen receptorwithin women's TMJ complex allows them to speculate that changes in metabolic function due to fluctuation in women's estrogen level can cause changes in flexibility of TMJ's ligament. ${ }^{6}$ These findings are thought to contribute to the increasing number of women who suffers from DDWR when compared to men.

It has been suggested that DDWR would be the first stage of disc displacement, and its progress to DDWoR, retrodiscitis or other intracapsular disorder is inevitable. However, such speculations is not consistent to all conditions and types of DDWR. Researchers have found that, from the study of 155 TMJ patient with DDWR,
$93.5 \%$ of them would not have this condition progresses; only $6.5 \%$ of these patients, or six TMJ, that progresses into DDWoR. The condition of DDWR can continueto remain unchanged as long as patient has an adaptive capacity to withstand clicking. The most common findings of adaptive capacity are in a form of retrodiscal fibrosis, where bilaminar zone of TMJ created fibrotic structures as an evidence of adaptive capacity of articular disc. This conditions can be stimulated through the use of occlusal splint (Fig 2). ${ }^{2,6,9}$
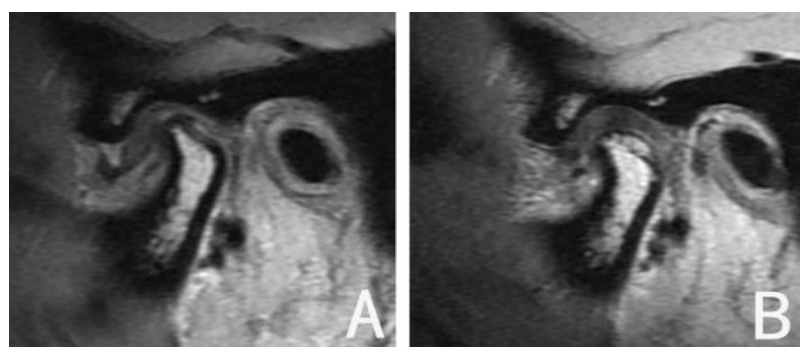

Figure 2A The condition of DDWR within TMJ complex before treatment, viewed using MRI; B TMJ complex after treatment with occlusal splint. Bone apposition can beseen in posterosuperior region of the condyle and the formation of retrodiscal fibrosis within the retrodiscal tissue. ${ }^{6}$

TMJ complex to confirm DDWR can be examined using MRI as the gold standard. . $^{2,5,8-10}$ MRI can accurately show the morphology and the articular disc position in relation to the bone structures within TMJ. The specificity value is around $88-90 \%$ with sensitivity value between 78 $83.3 \% .^{2,3,5}$ Severaltechniques available to analyze articular disc position in relation to condyle and fossa. One of the more common technique is the Draze-Enazmann method. Point $\mathrm{C}$ is designated as the centric point within the condyle head, while Point $D$ is the middle point within the posterior margin of posterior articular disc. Line 1 can then be created from point $C$ and be made perpendicular to Frankfort Horizontal plane. Line 2 can be made by joining point $C$ and $D$. when Line 1 and 2 are examined, the angle made between these two lines is known as disc-condyle angle. The normal value of this angle is between $-15^{\circ}$ and $+15^{\circ}$. When the value is larger than $+15^{\circ}$, then it is an indication of DDWR condition being positive (Fig 3). ${ }^{3,5}$

Ultrasonography can be used as an alternative way to examine the condition of DDWR. This technique has specificity value of $66.7 \%$ and sensitivity of $78.6 \%$. The drawbacks of USG is the lack of standardized examination technique, so it's accuracy varies in each examination when compared to $\mathrm{MRI}^{2}$ 


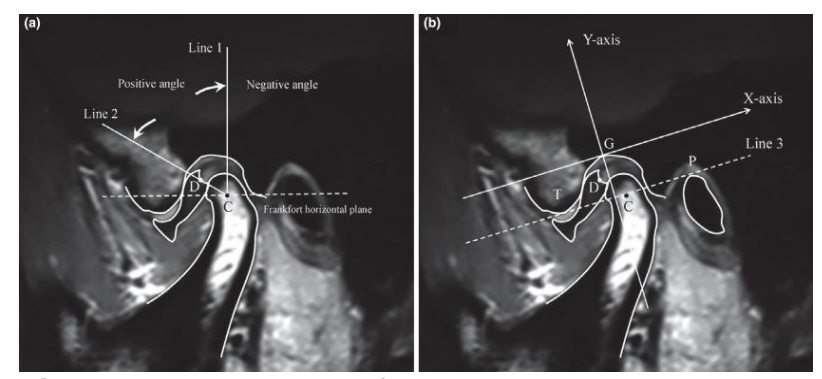

Figure 3AExamination ofDDWRusing MRI and DrazeEnazmann method. Point $C$ is the centric point of the condyle head, while point $D$ is the middle point of the posterior margin of articular disc. Line 1 can be made from point $\mathrm{C}$ and be made perpendicular to Frankfort Horizontal plane, while Line 2 is made by joining point $\mathrm{C}$ and $\mathrm{D}$. between Line 1 and 2 , it will create an angle known as disc-condyle angle; $\mathbf{B}$ coordinate measurement of articular disc position and condyle head. ${ }^{3,5}$

Aside from the advancement of technology, manual examination remains preferred examination method of choice is most TMJ dysfunction case. According to the Diagnostic Criteria for TMD (DC/TMD) ${ }^{11,12}$ examination of DDWR must meet at least one of thesecriteria: 1 ) in the last 30 days, any TMJ noise(s) present with jaw movement or function, or 2) patient report of any noise during the exam.

During the examination, patient must experience at least one of the following: 1) clicking, popping, and/or snapping noise during both opening and closing movement, detected with palpation during at least one of three repetitions of jaw opening and closing movement, or 2) clicking, popping and/orsnapping noise detected with palpation during at least one of three repetition of opening orclosing movement, and 3)clicking, popping, and/or snapping noise detected with palpation during at least one of three repetition of right or left lateral, or protrusive movement(s).

The use of imaging is the reference standard for this diagnosis. The use of panoramic imaging provides $34 \%$ sensitivity value and $92 \%$ specificity value. ${ }^{11,12}$ However, other research found that the methode proposed using DC/TMD only provides $44 \%$ sensitivity value and $46-57 \%$ specificity value in evaluating DDWR. ${ }^{2}$

According to Jeffrey Okeson, DDWR should have the following criteria: 1 ) there is a single joint sound during opening and closing movement in one or both TMJ. Joint sound does not include reciprocalsound orcrepitation sound;2) can have association with joint pain;3) normal mandibular motion, with maximum mouth opening more than $40 \mathrm{~mm}$ when measured from incisal edge of upper centralincisiveagainstlowercentral incisive tooth.

\section{Anterior repositioning splint}

Fabrication of ARS was first proposed by Farrar in $1970 .{ }^{3}$ The purpose of ARS is to return association between disc-condyle-fossa complex into normal position. When mandible is positioned more anterior dan maximum intercuspation (MI) by using ARS, this provides stability to TMJ. The use of reverse incline on ARS in the anterior maxillary region will provide guidance to occlude in a more anterior position (Fig 4). ${ }^{6}$
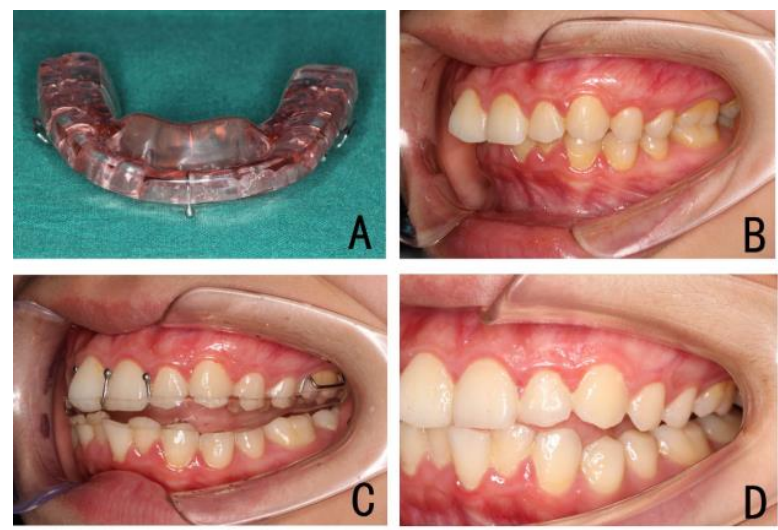

Figure 4AFull coverage ARS on anterior maxilla using the reverse incline plane bite block on palatal as guidance so that mandibula can be positioned more anterior; B-D occlusal re-establishment after the use of ARS. ${ }^{6}$

The treatment mechanism of ARS as a mode of therapy is still debatable. Two theories emerge that are generally acceptable among researcher. One of them is the 'recapturer' theory who stipulates that as condyle is placed more anteriorly, the relationshipbetween disc-condyle-fossaallows articular disc to recapture a normal relationship by guiding condyle to occlude along the posterior slope of articulareminence through periodic modification of ARS. With repeated use for short period of time, articular disc can stimulate bone appositionalong the posterosuperior area of condyle and/orcreating fibrotic structures along the retrodiscal tissue (figure 5). ${ }^{3,6,9}$ Another theory proposes that after the use of ARS, clinician must restore the whole dentition so that mandibula will return to therapeutic position, which is a position that locates condyle more anteriorly. This is known as the 'rebuilders' ${ }^{3}$ Okeson stated that the 'recapturer' theory is more acceptable because fibrotic structure along the retrodiscal tissue can remove pain symptoms that is associated with DDWR. Furthermore, mandibular position would be able to return back into a musculoskeletally stable position, when compared to rebuilders that maintains therapeutic position more anterior than muskuloskeletally stable position. ${ }^{1,9}$ 


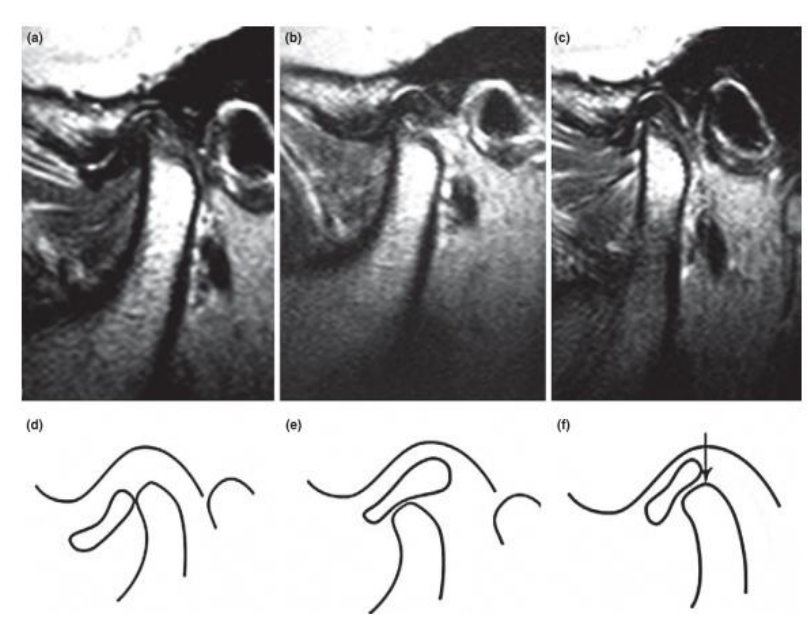

Figure 5 (A) DDWR before treatment. (B) DDWR after placement of ARS, where articular disc is recaptured to a more normal position in maximum intercuspation where loading of the joint is transferred within the thin intermediate zone between condyle and fossa. (C) Fibrotic structure is made as an adaptive response to the use of ARS after 6-8 weeks. Retrodiscal tissue does not elicit joint pain because of its fibrotic structure. (D-F) A diagrammatic representation of $A-C$, respectively. ${ }^{3}$

Primary indication of the use of ARS is to treatDDWR as well as disc displacement with intermittent locking on mandibula. The use of ARS has also been shown to be able to treat single clicking or reciprocal clicking joint that can be accompanied with pain. Patient with retrodiscitis can find some relief using ARS.

ARS commonly use hard acrylic and adapted on one of the jaws, usually the maxilla. Maxilla is the preferred choice because it is easier to make reverse incline ramp on a more stable occlusal surface when compared to mandible which is more mobile. A $2 \mathrm{~mm}$ thickness is often enough to provide an adequatestrength to the appliance. The incline ramp will guide mandible to a more anterior position. Appliance will be used and its efficacy will be evaluated. If symptoms do not improve, then patient's condition will be re-evaluated.

When ARS fails to relieve symptom, there is a possibility that the etiologic factor has notbeen addressed. Pain can come from TMJ complex, or better known as intracapsular pain, or it can also come from muscle, also known as intercapsular pain or myogenous pain. When pain comes from muscle, then symptom relieve can progress slowly, because of protective co-contraction muscle response that can cause uncomfortable muscle tension which can sensitize joint. Repeating diagnostic procedure becomes important for clinician to be able to better determine etiologic factor of each individual patient. ${ }^{1,9}$
Success rates is one factor that needs to be considered to determine the efficacy of the use of ARS. According to Okeson, joint sound cannot be the determining factor of ARS's success, because long term studies have indicated that more than $50 \%$ of patients afterARS treatment will have returning joint sound. Moreover, when factors that determine success includes the reduction of joint sound and pain resolution, the success rate of ARS treatment is only $28 \%$. However, if we modify the factors to only the resolution of pain, then the success rate increases into $75 \%$ over an observation period of $2.5-5$ years. ${ }^{13}$ Joint sound has been found to be more resistant to repair when compared to pain resolution after the use of ARS.

The use ofMRI has become the golden standard to evaluate articular disc position prior, during and after the insertion of ARS. This observation is to helpus determine whether joint sound can become the cause of DDWoR. The findings are summarized on table 1 , and it can be concluded that ARS can become a viable treatmenton joint pain to patients with DDWR, and joint sound does not determine the failure of ARS treatment; only $6 \%$ of patient with joint pain and DDWR that progresses into other internal derangementcondition such as DDWoRor joint locking. ${ }^{1-3,9}$ the use of ARS gives opportunity for retrodiscal tissue to adapt and form fibrotic structure to allow TMJ to function without joint pain while maintaining a more anterior positioned articular disc.

The use of ARS should meet the following criteria during insertion: 1) ARS must adapt well with the corresponding natural teeth so that retention and stabilization of splint can be maintained well. Retention should be examined by palpating the splint in patient's mouth;2) in a protruded mandible position, allmandibular teeth must contact evenly, where mandibular cusp have some contact with the occlusal surface of ARS; 3 ) the protruded position that is made from the splint must resolve symptoms during opening and closing of mouth at the new position; 4) during retruded mandible position, reverse incline ramp mustcontact the opposing teeth. As mandible elevates, splintmust guide mandible to therapeutic mandibular position; 5) ARS must be polished to have an even surface.

Post-insertion instruction varies among researcher; some pointed out that the use of ARS should be use for 24 hours, and only removed during eating and brushing teeth. Ma et al recommend creating a $5 \mathrm{~mm}$ thick ARS to remove reciprocal clicking, where occlusal grinding was per- 
formed to reduce ARS thickness $1 \mathrm{~mm}$ for every 4-6 weeks in the posterior region to induce vertical eruption of patient's teeth and achieve occlusal plane levelling. Patient willuseARS for 1-3 months, and deemed successful when joint clicking and pain did not return after 1-3 months post-treatment. ${ }^{6}$ According to Chen et al, the use of ARS should have at least 3 months of use for 24 hours, and continues by wearing ARS only at nighttime. ${ }^{3}$

Okeson suggest that the use of ARS should be limited on nighttime only. Daytime use reserves to condition where patientcannottolerate jointpain during working hours, and the use is limited to resolvingthepain only. When the pain resolves, the use of ARS should be discouraged, as to prevent changing mandible's position more anteriorly. In addition, patients were instructed with physical self-regulation (PSR) regimen to aid in TMJ treatment. The goal of PSRis to make patientconscious of theircondition, also known as cognitive awareness, in terms of spatial position of their mandible so that patient can reduce non-functional contact and excessive muscle activity actively to aid in TMD joint pain management. PSR involves pro-

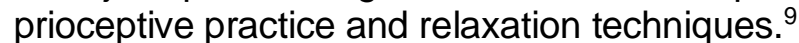

Treatment becomes tricky when patient's main complaint is a veryloud joint sound. For the majority of patients, patient's education is the treatment of choice. There is no standardized treatment of TMJ joint sound, so healthcare practitioner must perform conservative treatment as treatment of choice through education, PSR, occlusal splint, and muscle exercises of the jaw. Invasive treatmentis rarely indicated during DDWR because of the many risk associated with invasive surgical procedure.

When the use of ARS does not improve pain tolerance, healthcare workers need to consider two possibilities. One is that the adaptive process of retrodiscal tissue in inadequate in creating fibrotic structure to allow this tissue withstand functional load. In this case, the use of ARS must be lengthened to provide opportunity for the structure to adapt. However, if pain is caused by orthopedic instability, then patient may have returning symptoms after treatment with ARS. In this case, dental treatment needs to be administered so as to attain orthopedic stability. The treatment is seldom needed, however.

Invasive treatment can be considered when conservative treatmentdid not produce the intended result. Persistence of symptoms is one sign that indicates failure of ARS addressing the problem. Treatment options that can be considered are arthrocentesis, arthroscopy and surgical treatment. Arthrocentesis is a treatment of injecting therapeutic substance such as hyaluronate acid or corticosteroid with the intention to flush away algogenic substance that adheres in the articular disc, notable the superior joint space of the articular disc. Furthermore, the procedure also modifies intracranial pressure, which relieves pain. The use of arthrocentesis is indicated with closed lock TMJ, rheumatoid arthritis or adhesion. The use of arthrocentesis for DDWR is seldom indicated. ${ }^{14}$ Arthroscopy treatment uses arthroscope to directly observe superior joint space so as to identify and eliminate adhesion on joint. However, invasive treatment often is accompanied with elevated risk, such as extravasation of synovial fluid, facial nerve lesion, eye lesion, pre-auricular hematoma, intracranial perforation, and other risks. These risks needs to be addressed so that patient can receive for benefit having this treatment performed that the risk. ${ }^{9}$

It was concluded that the purpose of the treatment of DDWR should be to reduce intracapsular pain, and not necessarily returning articular disc position to a normal position. The use of ARS is another non-invasive treatment modality that can be offered to DDWR's patient so that the use of ARS will allow adaptive response by eliciting fibrotic structure on retrodiscal fossa and returning articular disc to a more normal position of disc-condyle-fossa. Variation exist regarding the post-treatment instruction, butmost afree that the use of ARS should only be on a short-term basis to reduce the risk of a more permanentunintended occlusal change. Adaptive response occurs between 8-10 weeks, and most patients experience relieve of pain. The lack of gold standard for the treatment of DDWR prompts healthcare provider to optforconservative treatment before considering advancing to a more invasive procedure.

\section{REFERENCES}

1. Okeson JP. Joint Intracapsular Disorders: Diagnostic and Nonsurgical Management Considerations. Dent Clin North Am. 2007;51(1):85-103.

2. Poluha RL, De La Torre Canales G, Costa YM, Grossmann E, Bonjardim LR, Conti PCR. Temporomandibular joint disc displacement with reduction: A review of mechanisms and clinical presentation. J Appl Oral Sci. 2019;27:1-9.

3. Chen HM, Liu MQ, Yap AUJ, Fu KY. Physiological effects of anterior repositioning splint on 
temporomandibular joint disc displacement: a quantitative analysis. J Oral Rehabil. 2017;44(9):664-672.

4. Chantaracherd $P$, John MT, Hodges JS, Schiffman EL. Temporomandibular joint disorders' impact on pain, function, and disability. J Dent Res. 2015;94(3):79S-86S.

5. Liu MQ, Lei J, Han JH, Yap AUJ, Fu KY. Metrical analysis of disc-condyle relation with different splint treatment positions in patients with TMJ disc displacement. J Appl Oral Sci. 2017;25(5):483-489.

6. Ma Z, Xie Q, Yang C, Zhang S, Shen Y, Abdelrehem A. Can anterior repositioning splint effectively treat temporomandibular joint disc displacement? Sci Rep. 2019;9(1):8-15.

7. Xie $Q$, Yang $C$, He D, et al. Will unilateral temporomandibular joint anterior disc displacement in teenagers lead to asymmetry of condyle and mandible? A longitudinal study. J Cranio-Maxillofacial Surg. 2016;44(5):590-596.

8. Litko M, Berger M, Szkutnik J, Różyło-Kalinowska I. Correlation between direction and severity of temporomandibular joint disc displacement and reduction ability during mouth opening. J Oral Rehabil. 2017;44(12):957-963.

9. Okeson JP. Management of Temporomandibular Disorders and Occlusion 8th Edition. 8th ed. Missouri: Elsevier Inc; 2020.

10. Kumar Rg, Gajapathi B, Ibrahim Mm, Karthik P, Rangarajan V, Yogesh P. Concepts of occlusion in prosthodontics: A literature review, part I. J Indian Prosthodont Soc. 2016;15(3):200.

11. Schiffman E, Look J, Anderson G, et al. DC/TMD for clinical and research application. 2014;28(1):6-27.

12. Okeson JP. Reliability and validity of the DC/TMD Axis I. J Oral Facial Pain Headache. 2018;32(1):19-21.

13. Okeson JP. long term treatment of disk interference disorders of the temporomandibular joint with anterior repositioning occlusal splints. :611-616.

14. Monje-Gil F, Nitzan D, González-Garcia R. Temporomandibular joint arthrocentesis. Review of the literature. Med Oral Patol Oral Cir Bucal. 2012;17(4). 